\title{
Blind Equalization Using Direct Channel Estimation
}

\author{
Hyung-Min Park ${ }^{1}$, Sang-Hoon $\mathrm{Oh}^{2}$, and Soo-Young Lee ${ }^{1}$ \\ 1 Department of BioSystems, and Brain Science Research Center \\ Korea Advanced Institute of Science and Technology \\ Daejeon, 305-701, Republic of Korea \\ \{hmpark, sylee\}@kaist.ac.kr \\ 2 Department of Information Communication Engineering \\ Mokwon University \\ Daejeon, 302-729, Republic of Korea \\ shoh@mokwon.ac.kr
}

\begin{abstract}
In performing blind equalization, we propose a direct channel estimation method based on entropy-maximization of input signal with its known probability density function. That is, the proposed method estimates filter coefficients of the channel instead of equalizing filter coefficients which most of equalization methods try to estimate. Because the channel usually has a much shorter length than the equalizing filter, this method requires much smaller parameters to be estimated, and the channel can be equalized with much less computational demands. In addition, simulation results show that the proposed method can recover signals with a much smaller error than conventional methods.
\end{abstract}

\section{Introduction}

Blind equalization has become an important research problem in digital signal processing because of its desirable features and the challenge it poses to researchers in the field. If a training sequence is available, an adaptive equalizer can be easily adapted using the standard least-mean-squares (LMS) algorithm. However, there are many cases such as high data rate, bandlimited digital communication systems where the transmission of a training sequence is impractical or very costly. Therefore, blind adaptive equalization algorithms that do not rely on training signals need to be developed.

Let us consider a single-input-single-output (SISO) discrete-time linear system, in which the relationship between the input and the output signal is given by

$$
x(n)=\sum_{k=0}^{L_{m}-1} h(k) s(n-k)+v(n) .
$$

The goal of blind equalization is to recover the input signal $s(n)$ from the output $x(n)$ without the assistance of a training sequence when the channel $h(k)$ is 
unknown. Typically, the input signal $s(n)$ is i.i.d., and the noise sequence $v(n)$ is modeled by a zero-mean white Gaussian noise process.

Many researchers have studied on the problem and proposed a number of blind equalization algorithms $[1,2]$. In most of blind equalization methods, a causal finite-impulse-response (FIR) filter as a linear equalizer is used to recover the input signal $s(n)$. Hence, the equalizer model can be formulated by

$$
u(n)=\sum_{k=0}^{L_{a}-1} w(k) x(n-k),
$$

where $w(k)$ is a filter coefficient of the equalizer. Since the blind equalization methods does not have a training sequence, adaptation of $w(k)$ usually makes use of some a priori statistical knowledge of the input signal $s(n)$.

In situations where the amplitude characteristics of $s(n)$ are roughly known, the class of Godard algorithms can be used [3]. Among the Godard algorithms, especially, the Sato algorithm and the constant modulus algorithm are very popular because of its simplicity [2]. If the probability density function of $s(n)$ is approximately known, an entropy-maximization algorithm for blind equalization can be derived by exploiting the higher order statistics (HOS) implicitly. It provides the same algorithm as the maximum likelihood estimation gives. Moreover, the signal is equalized with an improved convergence speed by applying the natural gradient to the blind equalization algorithm $[4-6]$. The entropy-maximization algorithm with the natural gradient is as follows [4]:

$$
\Delta w(k) \propto w(k)-\varphi\left(u\left(n-L_{a}+1\right)\right) r(n-k),
$$

where

$$
r(n)=\sum_{l=0}^{L_{a}-1} w\left(L_{a}-1-l\right) u(n-l) .
$$

However, the equalizer requires a much longer filter length than the channel $h(k)$ since the equalizing filter approximates the inverse of the channel. Therefore, the number of estimated parameters is also very large, and it requires somewhat heavy computational loads to update filter coefficients of the equalizer. In addition, a large number of parameters degrade the recovered signal after convergence.

In this paper, we propose an equalizing method based on estimating a channel directly instead of the equalizing filter. This method requires much smaller parameters to be estimated. Therefore, we can equalize the channel with much less computational complexity and provide the recovered signal with a much smaller error than the conventional equalizing methods which estimate the inverse of the channel.

\section{The Proposed Blind Equalization Algorithm}

For simple derivation, the SISO linear system (1) can be represented in $z$-domain as

$$
x(n)=H(z) s(n),
$$


where

$$
H(z)=\sum_{k=0}^{L_{m}-1} h(k) z^{-k} .
$$

In order to derive a new blind equalization algorithm, let us consider the input and the output signal of (5) over a $N$ sample block, defined by the following vectors:

$$
\begin{aligned}
S & =[s(0), s(1), \cdots, s(N-1)]^{T}, \\
X & =[x(0), x(1), \cdots, x(N-1)]^{T} .
\end{aligned}
$$

Both the input and the output signal, $s(n)$ and $x(n)$ are zeros for $n<0$.

Then, we can write the output signal vector $X$ as

$$
X=\left[\begin{array}{cccc}
h(0) & 0 & \cdots & 0 \\
h(1) & h(0) & \cdots & 0 \\
\vdots & \vdots & \ddots & \vdots \\
h(N-1) & h(N-2) & \cdots & h(0)
\end{array}\right] S
$$

Here, $h\left(L_{m}+1\right)=h\left(L_{m}+2\right)=\cdots=h(N-1)=0$ by assuming that the length of the channel, $L_{m}$ is much smaller than $N$.

The joint probability density of the output signal vector $X$ can be given by

$$
p(X)=\frac{p(S)}{\left|h(0)^{N}\right|},
$$

and $p(S)=p^{N}(s(n))$ for an i.i.d. input signal. Therefore, the log-likelihood of (9) is

$$
L(H(z))=-N \log |h(0)|+N \log p(s(n)) .
$$

An infinitesimal increment of the log-likelihood for an increment $d H(z)$ is

$$
d L(H(z))=L(H(z)+d H(z))-L(H(z)) .
$$

With the score function defined by

$$
\varphi(s(n))=-\frac{d}{d s(n)} \log p(s(n)),
$$

we have

$$
d \log p(s(n))=-\varphi(s(n)) d s(n),
$$

where $d s(n)$ is given in terms of $d H(z)$ as

$$
d s(n)=-H^{-1}(z) d H(z) s(n) .
$$

Define a modified differential $d M(z)$ as

$$
d M(z)=\sum_{k=-\infty}^{\infty} d m(k) z^{-k}=H^{-1}(z) d H(z) .
$$


Therefore,

$$
d \log p(s(n))=\varphi(s(n)) d M(z) s(n) .
$$

In the similar way, we can show that

$$
d \log |h(0)|=d m(0)
$$

Thus, substituting (16) and (17) into (10) and (11) gives

$$
d L(H(z))=-N d m(0)+N \varphi(s(n)) d M(z) s(n) .
$$

Maximizing the log-likelihood in terms of $d M(z)$ provides the following learning algorithm,

$$
\Delta M(z) \propto \frac{d L(H(z))}{d M(z)} .
$$

Using (15), the natural gradient algorithm for updating $h(k)$ is given by

$$
\begin{aligned}
\Delta h(k) & \propto H(z) \frac{d L(H(z))}{d m(k)} \\
& \propto-H(z) \delta(k)+H(z) \varphi(s(n)) s(n-k) \\
& =-h(k)+\varphi(s(n)) q_{k}(n),
\end{aligned}
$$

where

$$
q_{k}(n)=\sum_{l=0}^{L_{m}-1} h(l) s(n-k+l) .
$$

Note that the update of $h(k)$ depends on future values $s(n-k+l), k-l<0$. In addition, it involves very intensive computation to compute all $q_{k}(n), k=$ $0, \cdots, L_{m}-1$, at each time step. Practically, the algorithm is modified by introducing an $L_{m}-1$ sample delay to remove the non-causal terms and reusing past results assuming that $h(k)$ is not much changed over about $2 L_{m}-1$ time steps and $q_{k}(n) \approx q_{0}(n-k)$. Moreover, it is necessary to deal with complex-valued data for communication applications. With these considerations, the algorithm is modified as

$$
\Delta h(k) \propto-h(k)+\varphi\left(s\left(n-L_{m}+1\right)\right) q^{*}(n-k),
$$

where

$$
q(n)=\sum_{l=0}^{L_{m}-1} h^{*}\left(L_{m}-1-l\right) s(n-l) .
$$

Taking the additive white Gaussian noise into consideration, the recovered signal follows the Pearson mixture model which is a mixture of the normal distributions $[5,7]$. Therefore, $s(n)-\tanh (s(n))$ can be used for the score function $\varphi(s(n))$. To deal with complex-valued data in communication systems, the score function becomes

$$
\varphi(s(n))=\Re\{s(n)\}-\tanh (\Re\{s(n)\})+j[\Im\{s(n)\}-\tanh (\Im\{s(n)\})]
$$


since one can consider that the real part of the signal $s(n)$ is independent of the imaginary part.

After estimating the channel $h(k)$ with the proposed method, we can recover the input signal $s(n)$ as

$$
\hat{s}(n)=\sum_{k=0}^{L_{a}-1} \hat{h}^{-1}(k) x(n-k),
$$

where $\hat{s}(n)$ and $\hat{h}(k)$ denote estimations for $s(n)$ and $h(k)$, respectively. In order to compute $\hat{h}^{-1}(k)$, one can use lots of methods including [8]. In this paper, we adopt a method which makes use of reciprocal values of the results from the Fourier transform because of its simplicity.

\section{Computational Complexity}

In order to compare the computational loads of the proposed method and the conventional entropy-maximization method (3), let us consider the number of multiplications for complex-valued data. Assuming that the equalizing filter $w(n)$ has $L_{a}$ taps, the conventional method requires approximately $4 L_{a}$ multiplications for a sample. (Note that computational demands on the score function are negligible.) On the other hand, let us assume that the proposed method has $L_{m}$ taps for the estimated channel $\hat{h}(n)$ and $L_{a}$ taps for its inverse $\hat{h}^{-1}(n)$. In this case, the number of multiplications is approximately $3 L_{m}+2 L_{a}+L_{a} \log _{2} L_{a}$ using the fast Fourier transform.

When one performs adaptive learning, accumulating the update amounts over a sample block and updating the accumulation may often provide more stable convergence than 'one-by-one' updating. With the block updating, the conventional method requires about $3 L_{a}$ multiplications for a sample whereas about $L_{a}$ multiplications are required for a block. For the proposed method, the loads to compute $\hat{h}^{-1}(n)$, at most $L_{a}+L_{a} \log _{2} L_{a}$ multiplications, are imposed for each block. Therefore, about $2 L_{m}+L_{a}$ multiplications are required for a sample while the number of multiplications for a block is approximately $L_{m}+$ $L_{a}+L_{a} \log _{2} L_{a}$. Because a block usually contains a great many samples, the approximate ratio of the number of multiplications for the two methods, $R$ is

$$
R=\frac{2 L_{m}+L_{a}}{3 L_{a}}
$$

It is worthy of note that $L_{a}$ is much larger than $L_{m}$ since a typical FIR filter usually requires much larger taps for an approximated inverse filter. Therefore, the computational complexity can be considerably reduced by the proposed algorithm.

\section{Simulation Results}

We illustrate the performance of the proposed blind equalization algorithm via simulations. We have tested the algorithm with quadrature-amplitude-modulated 
(QAM) signals whose amplitudes are $\sqrt{2}$. Experimental results were compared in terms of the intersymbol interference (ISI). It can be computed as

$$
\operatorname{ISI}(d B)=10 \log \left(\frac{\sum_{k}|t(k)|^{2}-\max _{k}|t(k)|^{2}}{\max _{k}|t(k)|^{2}}\right),
$$

where $t(k)=\hat{h}^{-1}(k) * h(k)$ or $t(k)=w(k) * h(k)$. We have chosen the length of $\hat{h}^{-1}(k)$ to be the same as the length of $w(k)$.

The output signal $x(n)$ of the SISO linear system (1) was generated using an 8 tap non-minimum phase channel shown in Fig. 1 [9]. In order to equalize the channel, we have employed a 17 tap filter for $\hat{h}(n)$ with tap-centering initialization. $\hat{h}^{-1}(n)$ has been computed for 64 taps. The step size was 0.001 with 100 samples for a block. Fig. 2 shows the ISI for the proposed method without noise. For comparison, the simulation on the conventional method (3) has been performed, and the result was included. In this simulation, a 64 tap filter was used as the equalizing filter $w(n)$, and other parameters had the same values as in the proposed method. In addition, we also compared it with the well-known method proposed in [10]. In order to consider the effect of noise, we repeated the simulations for the corrupted signals, and Fig. 3 shows the result for the signal whose SNR was 10dB. From these figures, it can be easily seen that the proposed method has a much smaller error than the conventional methods.

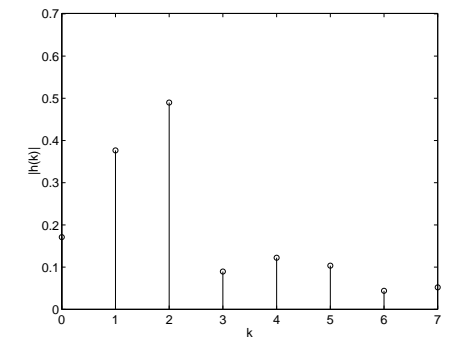

(a) magnitude

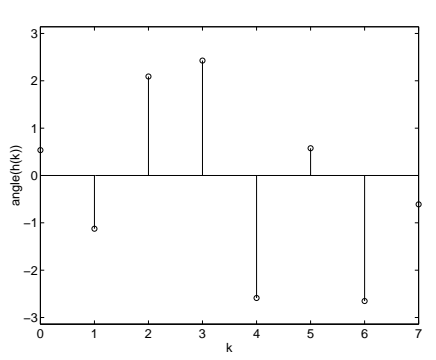

(b) phase

Fig. 1. A minimum phase channel.

\section{Conclusions and Further Works}

In this paper, we proposed a blind equalization method using direct channel estimation. Under the assumption that the probability density function of the input signal is known, we derived the channel estimation algorithm by maximizing the entropy of the signal. By estimating filter coefficients of the channel directly, 


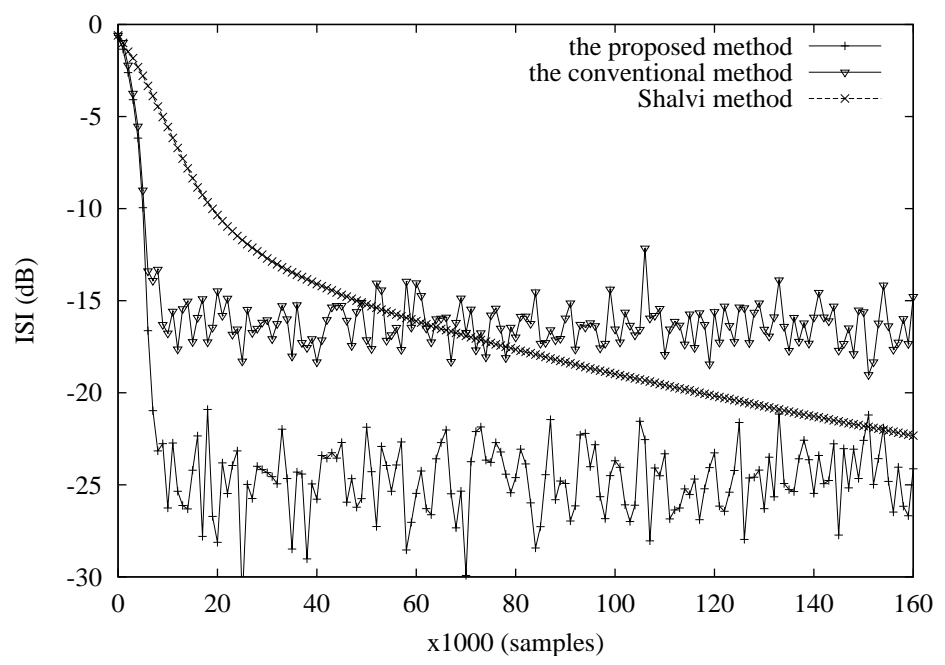

Fig. 2. ISI of the recovered signal without noise

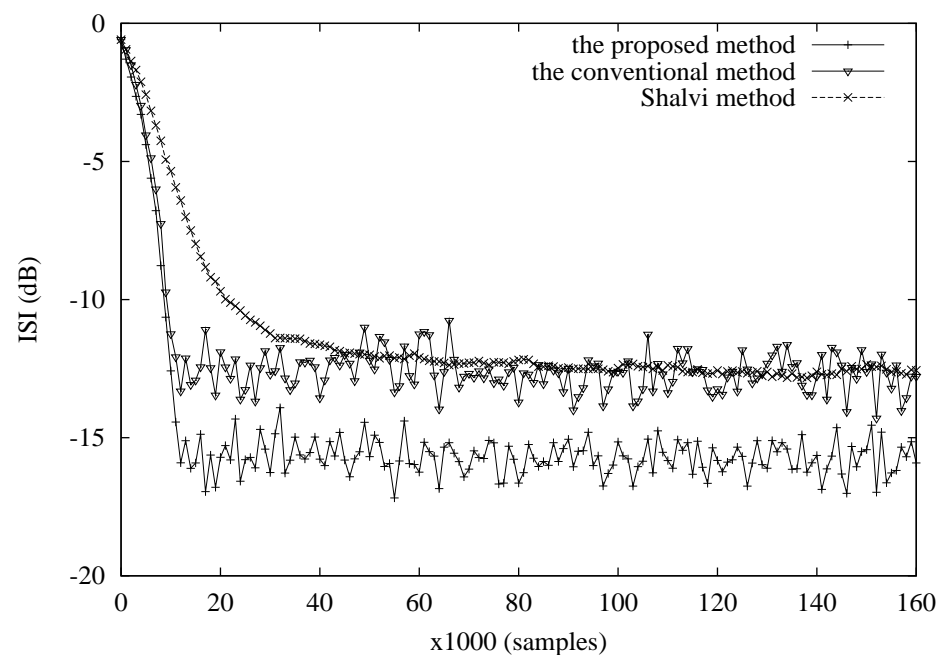

Fig. 3. ISI of the recovered signal with noise (10dB) 
the method has much smaller parameters to be estimated than the conventional method which estimates the equalizing filters. Therefore, the proposed method can equalize the channel with much less computational complexity. Moreover, the simulations indicated that a much smaller error was contained in the recovered signal with the proposed method than the conventional method. As some further works, now we try to apply the method to other applications and extend it to multi-channel deconvolution.

\section{Acknowledgment}

This work was supported by the Brain Neuroinformatics Research Program sponsored by Korean Ministry of Science and Technology.

\section{References}

1. Haykin, S. (ed.): Blind Deconvolution. Prentice Hall, Englewood, (1994)

2. Ding, Z., Li, Y. (eds.): Blind Equalization and Identification. Marcel Dekker, New York, (2001)

3. Godard, D.N.: Self-recovering Equalization and Carrier Tracking in Two Dimensional Data Communication Systems. IEEE Trans. Comm., 28 (1980) 1867-1875

4. Amari, S., Douglas, S.C., Cichocki, A., Yang, H.H.: Multichannel Blind Deconvolution and Equalization Using the Natural Gradient. Proc. IEEE Workshop on Signal Processing Advances in Wireless Comm., Paris, France, (1997) 109-112

5. Lee, T.-W.: Independent Component Analysis. Kluwer Academic Publishers, Boston, (1998)

6. Bell, A.J., Sejnowski, T.J.: An Information-Maximization Approach to Blind Separation and Blind Deconvolution. Neural Computation, 7 (1995) 1129-1159

7. Lee, T.-W., Girolami, M., Sejnowski, T.J.: Independent Component Analysis using an Extended Infomax Algorithm for Mixed Sub-Gaussian and Super-Gaussian Sources. Neural Computation, 11 (1999) 417-441

8. Radlović, B.D., Kennedy, R.A.: Nonminimum-Phase Equalization and Its Subjective Importance in Room Acoustics. IEEE Trans. Speech and Audio Processing, 8 (2000) $728-737$

9. IEEE Std 802.11a, Part11: Wireless LAN Medium Access Control (MAC) and Physical Layer (PHY) Specifications: High-Speed Physical Layer in the $5 \mathrm{GHz}$ Band, (1999)

10. Shalvi, O., Weinstein, E.: New Criteria for Blind Deconvolution of Nonminimum Phase Systems (Channels). IEEE Trans. Information Theory, 36 (1990) 312-321 